\section{Emissões otoacústicas por produto de distorção e audiometria tonal liminar: estudo da mudança temporária do limiar}

\author{
Silvana Frota ${ }^{1}$, \\ Maria Cecília Martinelli Iório ${ }^{2}$
}

\section{Distortion-product otoacoustic emissions and pure tone audiometry: a study of temporary threshold shifts}

Palavras-chave: audição, emissões otoacústicas, ruído, estimulação acústica.

Key words: hearing, otoacoustic emissions, noise, acoustic stimulation. \begin{abstract}
Resumo
$\mathbf{I}$ atualmente em uma das maiores causas de perdas auditivas neurossensoriais. Objetivo: O objetivo desse trabalho foi estudar os limiares de audibilidade e as emissões otoacústicas por produto de distorção, pré e pós-exposição a níveis elevados de ruído branco (100 dB NPS por 10 minutos), em função das variáveis lado da orelha e sexo, buscando informações para estabelecer a eficácia de ambos para detectar pequenas mudanças temporárias no limiar. Forma de estudo: prospectivo clínico randomizado. Material e método: Foram avaliados quarenta indivíduos otologicamente normais, sendo 20 do sexo masculino e 20 do sexo feminino, com idade variando de 18 a 36 anos. Ambos os testes, audiometria tonal e emissões otoacústicas por produto de distorção, foram realizadas de forma prévia e posterior à exposição ao ruído branco. Resultados: Os resultados mostraram que a audiometria tonal liminar é sensível para evidenciar mudanças temporárias nos limiares de audibilidade após exposição ao ruído branco nas freqüências de 2, 3, $4 \mathrm{KHz}$, independentemente, do lado da orelha e sexo, e que as emissões otoacústicas evidenciaram mudanças temporárias na sensibilidade auditiva após exposição ao ruído através da redução de suas amplitudes, nas freqüências de 2588 e $3614 \mathrm{~Hz}$ para o sexo feminino e nas freqüências de 932, 1304, 2588, $5128 \mathrm{~Hz}$ para o sexo masculino. Conclusão: Concluímos que tanto a audiometria tonal quanto as emissões otoacústicas evidenciaram sensibilidade para detectar mudanças temporárias significantes nos limiares de audibilidade e amplitudes, respectivamente, após a exposição ao ruído, variando de acordo com as freqüências estudadas.
\end{abstract}

Summary

\begin{abstract}
ntroduction: Noise-induced hearing loss is one of the most common causes for sensorineural hearing impairment. Objective: The purpose of this study was to evaluate pure tone and distortion product otoacoustic emissions (DPOAE) pre and post white noise exposition at high levels (100 dB SPL for 10 minutes), considering gender and ear variables, aiming at investigating pure tone audiometry and DPOAE efficiency in detecting subtle temporary threshold shifts (TTS). Study design: prospectivo clinical randomized. Material and method: Forty subjects, 20 male and 20 female ranging from 18 to 36 years old with no otological complaints were evaluated. Pure tone audiometry and DPOAE were carried out pre and post white noise exposure. Results: Pure tone audiometry was sensitive in detecting temporary threshold shifts after white noise exposition in 2,3 and $4 \mathrm{kHz}$, with no significant differences concerning gender and ear, whereas DPOAE revealed temporary shifts in audibility evidenced by amplitude reduction, in 2588 and 3614 in female subjects and in 932, 1304, 2588 and $5128 \mathrm{~Hz}$ in male subjects. Conclusion: We could conclude that either pure tone audiometry or DPOAE were sensitive in determining significant temporary shifts in hearing thresholds and amplitude, respectively, after white noise exposition, according to the involved frequency range
\end{abstract}

\footnotetext{
Fonoaudióloga. Mestre em Distúrbios da Comunicação Humana pela Universidade Federal de São Paulo - Escola Paulista de Medicina (UNIFESP-EPM), Professora Assistente de Audiologia da UFRJ.

${ }^{2}$ Fonoaudióloga. Doutora em Ciências dos Distúrbios da Comunicação Humana pela Universidade Federal de São Paulo - Escola Paulista de Medicina. Professora-Adjunto da Disciplina dos Distúrbios da Audição do Departamento de Otorrinolaringologia e Distúrbios da ComunicaçãoHumana da UNIFESP - EPM.

Tese apresentada à Universidade Federal de São Paulo - Escola Paulista de Medicina para a obtenção do Título de Mestre em Distúrbios da Comunicação Humana: Campo Fonoaudiológico.

Tese apresentada no XVI Encontro Internacional de Audiologia - Abril 2001 - Rio de Janeiro - RJ

Endereço para correspondência: Silvana Frota: Av. Niemeyer 925,Bl.2 apt $\square 1102$ - São Conrado - Rio de Janeiro - RJ - $22450-221$ - E-mail:frota@antares.com.br Artigo recebido em 10 de setembro de 2001. Artigo aceito em 20 de novembro de 2001.
} 


\section{INTRODUÇÃO}

O avanço tecnológico trouxe, além da desejada facilitação da vida moderna, um incremento substantivo de elementos estranhos e danosos ao organismo. O ruído é um dos agentes físicos resultantes dessas alterações ambientais, que ocorrem no âmbito do trabalho, dos transportes, do lazer e no convívio humano em geral.

A perda auditiva induzida por níveis elevados de pressão sonora consiste atualmente em uma das maiores causas de perdas auditivas neurossensoriais. Inúmeros fatores influenciam a ocorrência dessas perdas, destacandose o nível de pressão sonora, o tempo de exposição ao ruído, a intensidade e freqüência do ruído e a suscetibilidade individual.

Perdas auditivas por exposição ao ruído ocorrem, primeiramente, de forma reversível, através de mudanças temporárias de limiar na faixa de freqüências de 2000 a $6000 \mathrm{~Hz}^{3}$. As alterações temporárias do limiar de audibilidade vêm sendo amplamente estudadas, pois sua presença, em maior ou menor grau, sinaliza um prognóstico de suscetibilidade para perdas auditivas permanentes.

Estudos anatomopatológicos demonstram que na perda auditiva induzida por níveis elevados de pressão sonora existem alterações mecânicas e metabólicas localizadas principalmente nas células ciliadas externas do órgão de Corti, especialmente naquelas localizadas na porção basal da membrana basilar, por serem as mais sensíveis ${ }^{1,11,14,20,23}$.

É incontestável a importância que as Emissões Otoacústicas (OEA) vêm adquirindo nas últimas décadas, dentro do campo otológico. David Kemp ${ }^{12}$, em sua primeira publicação sobre o assunto, em 1978, descreveu a existência de um eco coclear, possível de ser mensurado no meato acústico externo. Após esse estudo, inúmeros outros se sucederam para aprimorar os conhecimentos acerca da existência do mecanismo ativo da cóclea, originado da biomecânica das células ciliadas externas (CCE) do Órgão de Corti.

As contrações rápidas e lentas conferem à cóclea as suas propriedades biomecânicas. Assim, a eletromotilidade das CCE gera uma energia mecânica, liberada durante a contração rápida, que segue, ciclo por ciclo, a freqüência de estimulação, até várias dezenas de $\mathrm{kHz}$, e funciona como um amplificador coclear capaz de afinada seletividade freqüencial.

Descritas no ano de 1979, por David Kemp ${ }^{13}$, as emissões otoacústicas por produto de distorção (DPOAE) são evocadas como resposta da intermodulação de dois tons puros simultâneos de freqüências próximas (F1 e F2), denominados tons primários, e apresentam como diferencial a capacidade de comportamento por freqüência. O teste das DPOAE caracteriza-se por ser um exame objetivo, rápido, não invasivo, indolor e de fácil aplicação.
Assim, alterações cocleares decorrentes da exposição a níveis elevados de pressão sonora devem provocar precocemente mudanças na amplitude das DPOAE, que são originadas no Órgão de Corti, pelas células ciliadas externas (CCE)

A realização dessa pesquisa teve como objetivo estudar os limiares de audibilidade e as emissões otoacústicas por produto de distorção, de forma prévia e de forma posterior à exposição a níveis elevados de ruído branco, buscando estabelecer a eficácia de ambos os testes para detectar pequenas mudanças temporárias no limiar.

\section{MATERIAL E MÉTODO}

A pesquisa foi realizada no Ambulatório de Audiologia Clínica, do Departamento de Otorrinolaringologia da Universidade Federal do Rio de Janeiro, UFRJ. Participaram da pesquisa 40 indivíduos otologicamente normais, sendo 20 do sexo feminino e 20 do sexo masculino, com idade variando de 18 a 36 anos. No total foram avaliadas 80 orelhas.

A Audiometria Tonal Liminar e as Emissões Otoacústicas Evocadas por Produto de Distorção foram realizadas em cabina acústica.

Foi utilizado um audiômetro da marca Interacoustics, modelo CE 10 padrão ANSI-69 e fone TDH - 39 coxim MX 41. Para o registro das emissões otoacústicas evocadas por produto de distorção foi utilizado o aparelho AuDX da marca BIO-LOGIC SYSTEMS CORP, acoplado a um computador 486 .

Os indivíduos foram submetidos às testagens de acordo com as seguintes etapas:

- Registro das emissões otoacústicas evocadas por produto de distorção e audiometria tonal liminar pré-exposição ao ruído;

- Exposição a um ruído branco (100 dB NPS) por um período de tempo de dez minutos;

- Registro das emissões otoacústicas evocadas por produto de distorção e audiometria tonal liminar após a exposição ao ruído.

O estímulo utilizado para evocar as DPOAE constituiu-se de dois tons puros de diferentes freqüências (F1 e F2), apresentados simultaneamente. A relação numérica entre as duas freqüências de estímulos F2/F1 é de aproximadamente 1.22. As intensidades de F1 e F2 foram respectivamente $65 \mathrm{~dB}$ NPS (L1) e $55 \mathrm{~dB}$ NPS (L2).

Nesse estudo analisamos a amplitude do produto de distorção em relação à freqüência sonora, que tem suas características demonstradas na Tabela 1, com os valores das freqüencias primárias F1 e F2, a média geométrica de F1 e F2, a proporção F2/F1 e o valor de 2 F1 - F2 (Tabela 1).

Foram analisadas as médias geométricas (GM) nas freqüências de 932, 1304, 1818, 2599, 3614 e de $5128 \mathrm{~Hz}$. 
As emissões otoacústicas por produto de distorção (PD) foram consideradas como presentes quando registradas acima do ruído de fundo (NF), gravadas a partir do ruído do próprio indivíduo e do sistema de registro (Figura 1).

A diferença resultante entre a amplitude do produto de distorção e o nível de ruído (PD - NF), foi o parâmetro escolhido para comparação e análise de amplitude pré e pós-estimulação acústica.

Foi realizado um estudo estatístico nas respostas da audiometria tonal limiar e das emissões otoacústicas, considerando as variáveis sexo e lado da orelha, utilizando os testes não-paramétricos de Wilcoxon e Mann-Whitney.

\section{RESULTADOS}

Ao compararmos a audiometria tonal liminar pré e pós-exposição ao ruído, verificamos que os limiares de audibilidade dos indivíduos de ambos os sexos apresentaram mudanças significantes, variando dentre as freqüências estudadas (Tabelas 2, 3, 4, 5 e 6). As maiores mudanças nos limiares de audibilidade (piora) ocorreram nas freqüências de 3000 (5,75 dBNA) e $4000 \mathrm{~Hz}$ (6,0 dBNA) em ambos os sexos.

Nas amplitudes das DPOAE, observamos que poucas permaneceram inalteradas após a estimulação ao ruído branco.

Como não foram observadas mudanças estatisticamente significantes entre as amplitudes das DPOAE nas orelhas direita e esquerda em ambos os sexos, os valores foram agrupados. Ao observamos os valores individuais

Tabela 1. Valores das freqüências primárias do estímulo nos produtos de distorção

\begin{tabular}{|c|c|c|c|c|}
\hline \multicolumn{2}{|c|}{ Primárias (Hz) } & \multirow{2}{*}{$\begin{array}{c}\text { Proporção } \\
\text { F2 / F1 }\end{array}$} & \multirow{2}{*}{$\begin{array}{c}\text { Média } \\
\text { geométrica } \\
\text { F1e F2 }(\mathrm{Hz})\end{array}$} & \multirow{2}{*}{$\begin{array}{c}\mathrm{DP} \\
\mathrm{F} 1-\mathrm{F} 2(\mathrm{~Hz})\end{array}$} \\
\hline F1 & F2 & & & \\
\hline 843 & 1031 & 1.22 & 932 & 656 \\
\hline 1171 & 1453 & 1.24 & 1304 & 890 \\
\hline 1640 & 2015 & 1.23 & 1818 & 1265 \\
\hline 2343 & 2858 & 1.22 & 2588 & 1827 \\
\hline 3280 & 3983 & 1.21 & 3614 & 2577 \\
\hline 4639 & 5670 & 1.22 & 5128 & 3608 \\
\hline
\end{tabular}

das amplitudes das DPOAE encontramos mudanças de amplitudes (piora) em todas as freqüências, porém em freqüências diferentes segundo a variável sexo. As maiores mudanças de amplitudes, em relação aos valores médios, foram observadas nas freqüências de $3614 \mathrm{~Hz}(2,41 \mathrm{~dB}$ NPS), nos indivíduos do sexo feminino, e $1304 \mathrm{~Hz}(2,5 \mathrm{~dB}$ NPS) para os indivíduos do sexo masculino (Tabela 7 ).

Para os indivíduos do sexo feminino encontramos diferenças estatisticamente significantes nas freqüências de 2588 e 3614 Hz e nos indivíduos do sexo masculino mudanças estatisticamente significantes nas amplitudes das emissões otoacústicas nas freqüências de 932, 1304, 2588, 5128 Hz (Tabela 8).

\section{DISCUSSÃO}

Em nosso estudo com indivíduos adultos audiologicamente normais, todos eles, independentemente das variáveis lado da orelha e sexo, apresentaram mudanças estatisticamente significantes na comparação dos limiares de audibilidade pré e pós-estimulação ao ruído branco, nas freqüências de 2000, 3000 e 4000 Hz.

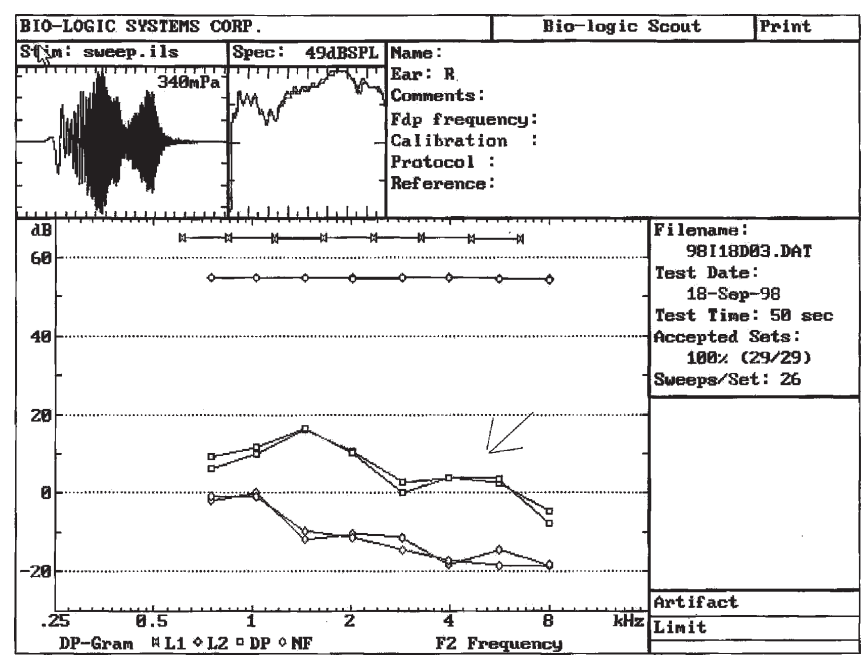

Figura 1. Representação das Emissões Otoacústicas por Produto de Distorção (PD), (indicadas pela seta), e do nível de ruído de fundo (NF), em cada freqüência testada.

- DP - Distortion product (Produto de distorção)

O - NF - Noise floor (Ruído de fundo)

Tabela 2. Limiares de audibilidade e medidas de tendência central - média, desvio padrão e mediana em dB NA, pré e pósexposição ao ruído, no sexo feminino, na orelha direita.

\begin{tabular}{|c|c|c|c|c|c|c|c|c|c|c|c|c|c|c|c|c|}
\hline \multirow{2}{*}{$\begin{array}{l}\text { Indivíduo } \\
\text { Feminino } \\
\end{array}$} & \multicolumn{2}{|c|}{$250 \mathrm{~Hz}$} & \multicolumn{2}{|c|}{$500 \mathrm{~Hz}$} & \multicolumn{2}{|c|}{$1 \mathrm{KHz}$} & \multicolumn{2}{|c|}{$2 \mathrm{KHz}$} & \multicolumn{2}{|c|}{$3 \mathrm{KHz}$} & \multicolumn{2}{|c|}{$4 \mathrm{KHz}$} & \multicolumn{2}{|c|}{$6 \mathrm{KHz}$} & \multicolumn{2}{|c|}{$8 \mathrm{KHz}$} \\
\hline & Pré & Pós & Pré & Pós & Pré & Pós & Pré & Pós & Pré & Pós & Pré & Pós & Pré & Pós & Pré & Pós \\
\hline Média & 10,25 & 11,50 & 8,75 & 9,50 & 3,50 & 6,50 & $-0,50$ & 4,50 & $-2,75$ & 3,00 & $-2,00$ & 2,75 & 2,00 & 5,00 & 3,25 & 4,25 \\
\hline DP & $\pm 5,49$ & $\pm 7,08$ & $\pm 5,09$ & $\pm 6,66$ & $\pm 4,61$ & $\pm 6,30$ & $\pm-2,5$ & $\pm 6,86$ & $\pm 4,99$ & $\pm 5,93$ & $\pm 4,10$ & $\pm 5,25$ & $\pm 6,56$ & $\pm 7,25$ & $\pm 6,34$ & $\pm 5,44$ \\
\hline
\end{tabular}


Tabela 3. Limiares de audibilidade e medidas de tendência central - média, desvio padrão e mediana em dB NA, pré e pósexposição ao ruído, no sexo feminino, na orelha esquerda.

\begin{tabular}{|c|c|c|c|c|c|c|c|c|c|c|c|c|c|c|c|c|}
\hline \multirow{2}{*}{$\begin{array}{l}\text { Indivíduo } \\
\text { Feminino }\end{array}$} & \multicolumn{2}{|c|}{$250 \mathrm{~Hz}$} & \multicolumn{2}{|c|}{$500 \mathrm{~Hz}$} & \multicolumn{2}{|c|}{$1 \mathrm{KHz}$} & \multicolumn{2}{|c|}{$2 \mathrm{KHz}$} & \multicolumn{2}{|c|}{$3 \mathrm{KHz}$} & \multicolumn{2}{|c|}{$4 \mathrm{KHz}$} & \multicolumn{2}{|c|}{$6 \mathrm{KHz}$} & \multicolumn{2}{|c|}{$8 \mathrm{KHz}$} \\
\hline & Pré & Pós & Pré & Pós & Pré & Pós & Pré & Pós & Pré & Pós & Pré & Pós & Pré & Pós & Pré & Pós \\
\hline Média & 8,75 & 12,00 & 6,50 & 7,75 & 1,50 & 4,00 & $-0,75$ & 4,50 & $-2,25$ & 1,75 & $-1,75$ & 4,25 & 7,75 & 10,50 & 4,25 & 5,50 \\
\hline DP & $\pm 8,40$ & $\pm 6,76$ & $+5,87$ & $\pm 6,58$ & $+4,61$ & $+5,52$ & $\pm 7,12$ & $\pm 9,16$ & $+5,72$ & $\pm 8,62$ & $\pm 5,44$ & $\pm 6,12$ & $\pm 8,34$ & $\pm 8,41$ & $\pm 10,03$ & $\pm 11,57$ \\
\hline
\end{tabular}

Tabela 4. Limiares de audibilidade e medidas de tendências central - média, desvio padrão e mediana em dB NA, pré e pósexposição ao ruído, no sexo masculino, na orelha direita

\begin{tabular}{|c|c|c|c|c|c|c|c|c|c|c|c|c|c|c|c|c|}
\hline \multirow{2}{*}{$\begin{array}{l}\text { Indivíduo } \\
\text { Masculino }\end{array}$} & \multicolumn{2}{|c|}{$250 \mathrm{~Hz}$} & \multicolumn{2}{|c|}{$500 \mathrm{~Hz}$} & \multicolumn{2}{|c|}{$1 \mathrm{KHz}$} & \multicolumn{2}{|c|}{$2 \mathrm{KHz}$} & \multicolumn{2}{|c|}{$3 \mathrm{KHz}$} & \multicolumn{2}{|c|}{$4 \mathrm{KHz}$} & \multicolumn{2}{|c|}{$6 \mathrm{KHz}$} & \multicolumn{2}{|c|}{$8 \mathrm{KHz}$} \\
\hline & Pré & Pós & Pré & Pós & Pré & Pós & Pré & Pós & Pré & Pós & Pré & Pós & Pré & Pós & Pré & Pós \\
\hline Média & 11,75 & 11,00 & ,00 & 11,00 & 9,00 & 11,00 & 5,2 & 9,00 & 7,25 & 11,50 & 5,75 & 9,25 & 10,25 & 11,50 & 5,00 & 8,00 \\
\hline & $\pm 5,19$ & $\pm 5,52$ & $\pm 5,38$ & $\pm 4,16$ & $\pm 6,40$ & $\pm 7,12$ & $\pm 5,72$ & $\pm 5,75$ & $\pm 8,02$ & $\pm 9,74$ & $\pm 10,58$ & $\pm 11,61$ & $\pm 8,65$ & $\pm 7,79$ & $\pm 9,38$ & $\pm 10,68$ \\
\hline
\end{tabular}

Tabela 5. Limiares de audibilidade e medidas de tendência central - média, desvio padrão e mediana em dB NA, pré e pósexposição ao ruído, no sexo masculino na orelha esquerda

\begin{tabular}{|c|c|c|c|c|c|c|c|c|c|c|c|c|c|c|c|c|}
\hline \multirow{2}{*}{$\begin{array}{l}\text { Indivíduo } \\
\text { Masculino } \\
\end{array}$} & \multicolumn{2}{|c|}{$250 \mathrm{~Hz}$} & \multicolumn{2}{|c|}{$500 \mathrm{~Hz}$} & \multicolumn{2}{|c|}{$1 \mathrm{KHz}$} & \multicolumn{2}{|c|}{$2 \mathrm{KHz}$} & \multicolumn{2}{|c|}{$3 \mathrm{KHz}$} & \multicolumn{2}{|c|}{$4 \mathrm{KHz}$} & \multicolumn{2}{|c|}{$6 \mathrm{KHz}$} & \multicolumn{2}{|c|}{$8 \mathrm{KHz}$} \\
\hline & Pré & Pós & Pré & Pós & Pré & Pós & Pré & Pós & Pré & Pós & Pré & Pós & Pré & Pós & Pré & Pós \\
\hline Média & 11,00 & 10,75 & 8,25 & 7,75 & 8,25 & 11,75 & 6,0 & 10,25 & 5,50 & 10,50 & 6,00 & 12,50 & 11,25 & 14,50 & 8,00 & 10,00 \\
\hline DP & $\pm 5,28$ & $\pm 5,19$ & $\pm 4,66$ & $\pm 4,43$ & $\pm 7,12$ & $\pm 7,30$ & $\pm 8,36$ & $\pm 8,50$ & $\pm 8,41$ & $\pm 7,93$ & $\pm 10,74$ & $\pm 10,57$ & $\pm 8,44$ & $\pm 7,23$ & $\pm 9,36$ & $\pm 9,73$ \\
\hline
\end{tabular}

Tabela 6. Valores de p calculado da estatística de Wilcoxon, dos limiares de audibilidade pré e pós-exposição ao ruído, Segundo a variável lado da orelha e Sexo

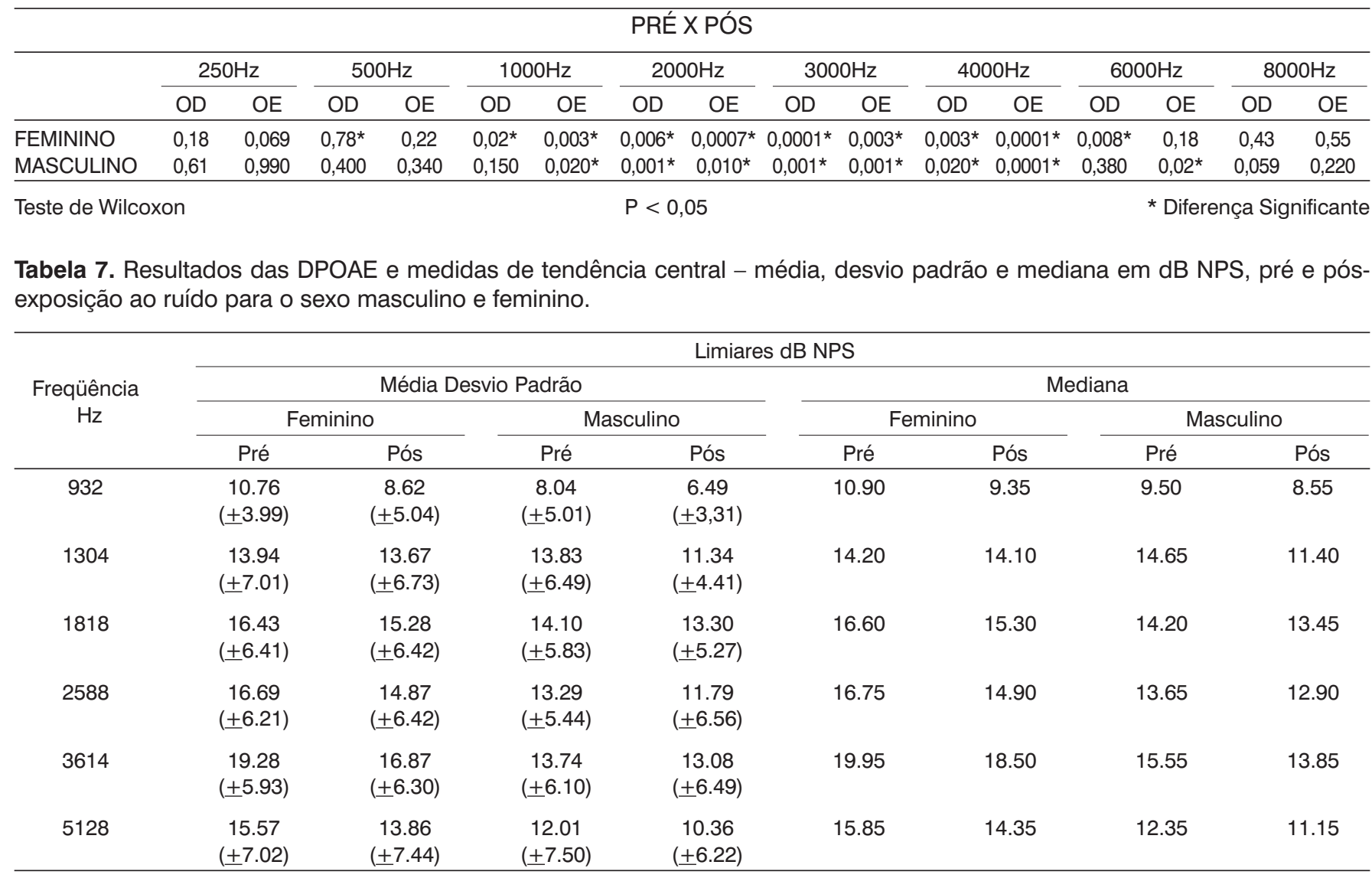


Tabela 8. Resultados do teste de Wilcoxon, das DPOAE pré e pós-exposição ao ruído, para os sexos feminino e masculino.

\begin{tabular}{lcccccc}
\hline & \multicolumn{6}{c}{ PRÉ X PÓS } \\
\cline { 2 - 7 } & $932 \mathrm{~Hz}$ & $1304 \mathrm{~Hz}$ & $1818 \mathrm{~Hz}$ & $2588 \mathrm{~Hz}$ & $3614 \mathrm{~Hz}$ & $5128 \mathrm{~Hz}$ \\
\hline Feminino & 0,215 & 0,862 & 0,317 & $0,009^{*}$ & $0,002^{*}$ & 0,092 \\
Masculino & $0,002^{*}$ & $0,0046^{*}$ & 0,328 & $0,044^{*}$ & 0,613 & $0,039^{\star}$ \\
\hline
\end{tabular}

Teste de Wilcoxon $\quad \mathrm{P}<0,05$

* Diferença Significante

Outros autores ${ }^{3,4,18,21,22}$ também estudaram a mudança do limiar de audibilidade após exposição ao ruído e encontraram concordância no que diz respeito às alterações de altas freqüências.

Em relação às emissões otoacústicas, inúmeros estudos ${ }^{9}, 10,15,16,17,24$ referem haver uma alteração nas amplitudes das emissões otoacústicas (piora) após exposição ao ruído. Estes estudos divergem nas freqüências alteradas, talvez em função da diferença entre a metodologia adotada.

Outro estudo ${ }^{15}$ mostrou diferenças significantes de $20 \mathrm{~dB}$ ou mais entre as amplitudes das emissões evocadas por produto de distorção das orelhas direita e esquerda em apenas 4KHz. Análises das amplitudes das curvas demonstraram que as mulheres apresentaram limiares mais baixos em torno de $10 \mathrm{~dB}$.

Em nosso estudo, não houve diferenças estatisticamente significantes entre as amplitudes das DPOAE das orelhas direita e esquerda, sendo possível agrupá-las. Porém, quando realizamos o estudo segundo a variável sexo, encontramos diferenças significantes na freqüência de $3614 \mathrm{~Hz}$.

Utilizando protocolos onde L1 era diferente de L2 $2^{24}$, a sensibilidade para detectar diferenças de amplitudes pré e pós-exposição a ruído (105 dB NPS centrado na freqüência de $2800 \mathrm{~Hz}$ ) era maior, sendo esta diferença de 5 a 6 dB. Em estudo paralelo foi referido que a redução da amplitude do DPOAE está relacionada, não linearmente, à diminuição do limiar de audibilidade. Para cada $1 \mathrm{~dB}$ NPS de decréscimo na amplitude das DPOAE o autor encontrou $2 \mathrm{~dB}$ nos limiares tonais. Os autores concluem serem importantes as diferenças entre L1 e L2, pois evidenciaram maior sensibilidade para captar alterações das CCE.

Estudo de um grupo de cobaias 5 expostos a um ruído de $80 \mathrm{~dB}$ NPS de meia oitava de banda em $6000 \mathrm{~Hz}$, por quatro horas de exposição cujo parâmetros de estímulos usados para evocar as DPOAE foram de F2/F1 e L2=L1-10, apresentaram como resultado a atenuação das emissões, a qual foi máxima na freqüência de meia oitava de banda da exposição. Utilizando os mesmos parâmetros, a experiência foi repetida a $90 \mathrm{~dB}$ NPS e resultou em um déficit das emissões, principalmente nas altas freqüências, que foram recuperadas após oito dias da exposição, enquanto na primeira a recuperação se deu dois dias após a exposição.
Ainda estudando cobaias, outro autor ${ }^{6}$ submeteu à exposição ao ruído, por dez dias consecutivos, dois grupos diferentes de cobaias: as acostumadas e as não acostumadas a estímulos sonoros. As emissões otoacústicas por produto de distorção foram utilizadas para monitoramento da perda auditiva induzida por exposição ao ruído. Como resultado foi observado que o grupo acostumado a sons foi significantemente menos afetado pela super estimulação durante os dias iniciais de exposição a ruídos, comparando com o grupo não acostumado a sons. As cobaias acostumadas a sons tornaram-se gradualmente mais afetadas pela super estimulação, nas sessões diárias subseqüentes.

Outro experimento ${ }^{7}$ utilizou, dentre outros testes, as DPOAE com o objetivo de pesquisar a alteração temporária da função coclear. Para tanto foi utilizado um ruído de 102 dB NPS centrado na freqüência de $2000 \mathrm{~Hz}$. A magnitude do efeito da exposição ao ruído foi vista pela amplitude das emissões otoacústicas por produto de distorção entre 2000 a $4000 \mathrm{~Hz}$. Como resultado, após a exposição ao ruído, a diminuição da média das amplitudes das DPOAE nas freqüências de 2000 a $4000 \mathrm{~Hz}$ (sendo a de $3000 \mathrm{~Hz}$ a mais afetada) foi mais significativa, medidas no primeiro minuto $(14,7 \mathrm{~dB})$ comparada com a redução das amplitudes após dois minutos que foi $6,8 \mathrm{~dB}$ e em vinte e dois minutos que foi de 4,1 $\mathrm{dB}$. Os autores concluíram que o TTS foi maior no primeiro minuto e que não houve nenhuma relação entre o TTS e a amplitude dos DPOAE após vinte e dois minutos de exposição.

Também foi observado ${ }^{8}$ redução maior na amplitude das DPOAE, em aproximadamente meia oitava na freqüência do ruído. Este grau da redução da amplitude foi maior quando estímulos de sons de baixa freqüência foram utilizados.

No registro das mudanças de amplitudes das DPOAE após exposição a $90 \mathrm{~dB} \mathrm{NPS}^{2}$, foi verificado uma diminuição de até $5 \mathrm{~dB}$ nessas amplitudes após a exposição.

Estudos recentes ${ }^{19}$ medindo o limiar das DPOAE em 102 orelhas de indivíduos otologicamente normais, antes e depois da exposição ao ruído, registraram mudança temporária de limiar, imediatamente após o ruído, principalmente em $4000 \mathrm{~Hz}$. Essas reduções de amplitude oscilaram de 2 a $2,5 \mathrm{~dB}$, em uma freqüência que variou de 2000 a 5000 Hz. Os autores observaram uma recuperação das amplitudes com o passar do tempo.

Um estudo comparativo ${ }^{9}$ entre a audiometria tonal e as emissões otoacústicas por produto de distorção em 450 trabalhadores, da linha de produção de uma metalúrgica, expostos a níveis de ruído variados, mostrou que as emissões otoacústicas em indivíduos expostos ao ruído caracteriza-se pelo acometimento principalmente das freqüências altas. Essa alteração progride com redução da intensidade ou da amplitude do produto de distorção e com o aumento do espectro de freqüências acometidas em direção às médias e baixas freqüências. Foi também 
constatado pelos autores que existe uma relação inversa entre audiometria e otoemissão, ou seja, conforme aumenta o limiar audiométrico diminui a amplitude do produto de distorção. Para os autores, a otoemissão por produto de distorção não substitui a audiometria tonal.

Em relação às emissões otoacústicas, tornou-se muito mais difícil a comparação do nosso trabalho com outros autores, uma vez que os parâmetros empregados para a pesquisa dos DPOAE, L1 e L2 como também F1 e F2 podem ser escolhidos aleatoriamente.

Outro fator a ser considerado, e que também dificultou a comparação dos nossos resultados com os resultados encontrados na literatura, está relacionado à exposição ao ruído, no que diz respeito ao tipo e espectro do ruído e ao tempo de exposição.

Como ponto de concordância, encontramos em todos os trabalhos consultados, independentemente do critério adotado, uma modificação (diminuição) na amplitude das emissões otoacústicas, variando de acordo com a freqüência utilizada.

\section{CONCLUSÃO}

A partir da análise crítica dos resultados pudemos estabelecer as seguintes conclusões:

I- A Audiometria Tonal Liminar mostrou-se sensível para evidenciar mudanças temporárias nos limiares de audibilidade após exposição ao ruído branco nas freqüências de 2000, 3000 e 4000 Hz, independentemente, do lado da orelha e sexo;

II- A Audiometria Tonal Liminar também demonstrou mudanças temporárias nos limiares de audibilidade estatisticamente significantes, após exposição ao ruído branco, nas freqüência de $1000 \mathrm{~Hz}$ (exceto para o sexo masculino à direita) e de $6000 \mathrm{~Hz}$ para o sexo feminino à direita, e para o sexo masculino à esquerda;

III- A Emissão Otoacústica por Produto de Distorção evidenciou mudanças temporárias na sensibilidade auditiva através da redução estatisticamente significante de suas amplitudes, após a exposição ao ruído branco, nas freqüências de 2588 e $3614 \mathrm{~Hz}$ para os indivíduos do sexo feminino e nas freqüências de 932, 1304, 2588 e $5128 \mathrm{~Hz}$ para os indivíduos do sexo masculino;

Ambos os testes aplicados nessa pesquisa, Audiometria Tonal Liminar e Emissão Otoacústica por Produto de Distorção, evidenciaram sensibilidade para detectar mudanças temporárias estatisticamente significantes nos limiares de audibilidade e amplitudes, respectivamente, após a exposição ao ruído branco.

\section{REFERÊNCIAS BIBLIOGRÁFICAS}

1. ALBERNAZ, P.L.M.; COVELL, W.P. - Acoustic trauma lesions by fluorescence microscopy. Laryngoscope, 72:1278-96, 1962.
2. ATTIAS, J.; BRESLOFF, I. - Noise induced temporaly otoacoustic emission shifts. J. Basic Clin. Physiol., 7(3):221-33,1996.

3. AZEVEDO, A.P.; OKAMOTO, V.A.; BERNARDI, R.C. - Considerações sobre Ruído. In: COSTA, D.F.; CARMO, J.C.; SETTIMI, M.M.; SANTOS U.P. Programa de Saúde dos Trabalhadores. São Paulo, Hucitec, 1989.

4. BUNCH, C.C. - The diagnosis of occupational or traumatic deafness. A historical and audiometry study. Laryngoscope, 47:615-91, 1937.

5. CHANG, K.W.; NORTON, S.J. - The Effects of Continuous Versus Interrupted Noise Exposures on Distortion Product Otoacoustic Emission in Guinea Pigs. Hear. Res., 96 (1-2):1-12, 1996.

6. DAGLI, S.; CANLON, B. - The Effect of Repeated Daily Noise Exposure on Sound - Conditioned and Unconditioned Guinea Pigs. Hear. Res, 104 (1-2):39-46, 1997.

7. ENGDAHL, B. - Effects of Noise and exercise on Distortion Product Otoacoustic Emissions. Hearing Research, 93:72-82, 1996.

8. ENGDAHL, B.; KEMP, D.T. - The Effect of Noise Exposure on the Details of Distortion Product Otoacoustic Emissions in Humans. $J$ Soc. Am., 99:1573-87, 1996.

9. FUKUdA, C.; MUNHOZ, M.S.L.; TOLEDO, F.B.; HASSAN, E.S. Emissões otoacústicas por produto de distorção em trabalhadores expostos a ruído. Acta Awho, 17 (4):176-85,1998.

10. GORGA,M.P.; NEELY, S.T.; BERGMAN, B.; BEAUCHAINE, K.L.; KAMINSKI, J.R.; PETERS, J.; JESTEADT, W. - Otoacoustic emissions from normal-hearing and hearing-impaired subjects: distortion product responses. J. Acoust. Soc. Am., 93(2):2050-60,1993.

11. INGRASIAS M.; SCHUKNECHT, H.F.; MYERS, E.N. - Cochlear pathology in humans with stimulation deafness. The Journal of Laryngology and Otology, 78:115-23, 1964.

12. KEMP, D.T. - Stimulated acoustic emission from within the human auditory System. J. Acoustic. Soc. Am., 64(5):1386-91,1978.

13. 13. KEMP, D.T. - Evidence of mechanical nonlinearity and frequency selective wave amplification in the cochlea. Arch. Oto-Rhino-Laryngol., 224:37-45,1979.

14. LIM, D.J.; MEINICK. - Acoustic damage of the cochlea; A scanning and tranmission electron microscopic observation. Arch. Otolaryng., 94:294, 1971.

15. LONSBURY-MARTIN, B.L.; HARRIS, F.P.; STAGNER, B.B.; HAWKINS, M. D.; MARTIN, G.K. - Distortion product emissions in humans. Basic properties in normaly hearing subjects. Ann. Otol. Rhinol. Laryngol., 147:3-14, 1990. Suppl. 99.

16. LOPES, O.; CARLOS, R. - Produto de Distorção das Emissões Otoacústicas. Rev. Bras. Med. Otorrinolaringol.,3(5):224-37, 1996.

17. LOPES, O.; CARLOS, R. - Emissões Otoacústicas. In: LOPES FILHO, O. Tratado de Fonoaudiologia. São Paulo, Roca. p.221-237, 1997

18. MIRANDA, C.R., DIAS, C.R. - Perda Auditiva Induzida pelo Ruído em Trabalhadores em Bandas e Trios Elétricos de Salvador, Bahia. Rev Bras. Otorrinolaringol, 64(5), 1999.

19. OEKEN, J.; MENZ, D. - Amplitude changes in distorcion products of otoacoustic emission after acute noise exposure. Laryngorbinootologie, 75(5):265-9, 1996.

20. OLIVEIRA, J. - Fisiologia clínica da Audição. In: NUDELMANN, A. A.; COSTA, E. A.; SELIGMAN, J.; IBAÑEZ, R.N. Perda Auditiva Induzida por Ruído. Porto Alegre, Bagagem. p.101-42, 1997.

21. SANTOS, U.P.; COSTA, D. F.; CARMO, J.C. - Programa de Conservação Auditiva em Trabalhadores Expostos à Ruído. In: COSTA, D.F.; CARMO, J.C.; SETTIMI, M.M.; SANTOS, U.P. Programa de Saúde de Saúde dos Trabalhadores. São Paulo, Hucitec. p.125-55, 1989.

22. RUSSO, I.C.P.; SANTOS, T.M.M.; BUSGAIB, B.B.; OSTERNE, F.J.V. Um Estudo Comparativo Sobre os Efeitos da Exposição à Música de Trio Elétrico. Rev. Bras. Otorrinolaringol, 61(6):477-84, 1995.

23. SULKOWSKI, W.J. - Industrial Noise Pollution and Hearing Impairment Springfield, US Department of Commerce. 1980. National Technical Information Service.

24. SUTTON, L.A.; LONSBURY-MARTIN, B.L.; MARTIN, G.K; WHITEHEAD, M.L. - Sensitivity of distortion-product otoacoustic emissions in humans to tonal over-exposure: Time course of recovery and effects of lowering L2. Hearing Research, 75:161-74, 1994. 